\title{
Accumulation of AGEs and VEGF in Eyes of SDT Rats
}

\author{
Fumihiko Toyoda $^{1}$, Akihiro Kakehashi ${ }^{*}, 1$, Kana Hashimoto ${ }^{1}$, Nozomi Kinoshita ${ }^{1}$, Chiho Kanbara ${ }^{1}$, \\ Hiroko Yamagami ${ }^{1}$, Hiroyuki Tamemoto ${ }^{2}$, San-e Ishikawa ${ }^{2}$, Yoh Dobashi ${ }^{2}$, Masanobu Kawakami ${ }^{2}$ \\ and Yasunori Kanazawa ${ }^{3}$
}

\author{
${ }^{I}$ Department of Ophthalmology, Jichi Medical University, Saitama Medical Center; Saitama, Japan \\ ${ }^{2}$ Department of Integrated Medicine I, Jichi Medical University, Saitama Medical Center, Saitama, Japan \\ ${ }^{3}$ Japanese Diabetes Foundation, Tokyo, Japan
}

\begin{abstract}
Background/Aims: The Spontaneously Diabetic Torii (SDT) rat develops advanced diabetic retinopathy (DR). The aim of this study was to identify advanced glycation end products (AGEs) related to vascular endothelial growth factor (VEGF) expression, a cause of DR in SDT rats.

Methods: One eye was obtained from six SDT rats (blood glucose, $>250 \mathrm{mg} / \mathrm{dl}$ ) and 10 nondiabetic normal SpragueDawley (SD) rats and prepared for immunohistochemical study of VEGF and AGEs (pyrraline, pentosidine, carboxy methyl lysine $[\mathrm{CML}])$. Immunostaining was described as minimal, moderate, and severe.

Results: In diabetic rats, for CML, five eyes had severe and one moderate immunostaining. For pyrraline, one eye had moderate and five eyes minimal immunostaining. For pentosidine, one eye had moderate and five eyes minimal immunostaining. For VEGF, three eyes each had moderate and severe immunostaining. In nondiabetic rats, for CML one eye had minimal, seven had moderate, and two had severe immunostaining. For pyrraline, four eyes had moderate and six eyes minimal immunostaining. For pentosidine, 10 eyes had minimal immunostaining. For VEGF, one eye had moderate and nine had minimal immunostaining. The prevalence rates of CML and VEGF were significantly $(\mathrm{P}<0.05, \mathrm{P}<0.001$, respectively) greater in diabetic than in nondiabetic rats. The prevalence rates of pyrraline and pentosidine were not significantly $(\mathrm{P}=0.35, \mathrm{P}=0.38)$ different between diabetic and nondiabetic rats.

Conclusion: CML coexists with VEGF and may be involved in the pathogenesis of severe ocular complications in SDT rats.
\end{abstract}

Keywords: Diabetes, diabetic retinopathy, advanced glycation end products (AGEs), vascular endothelial growth factor (VEGF).

\section{INTRODUCTION}

Diabetic ocular complications, such as diabetic retinopathy (DR), cataracts, and rubeotic glaucoma, impair vision and quality of life. DR, one of the most serious complications of diabetes mellitus, frequently leads to blindness [1-3]. Vascular endothelial growth factor (VEGF), an important cytokine that induces proliferative DR [4,5], is correlated with several metabolic changes, including increased polyol pathway activity [6-9], activation of protein kinase C (PKC) [10-14], increased oxidative stress $[13,15,16]$, and accumulation of advanced glycation end products (AGEs) [13,17-19]. These induce retinal vascular dysfunction and retinal ischemia. New drugs targeting these biochemical changes, such as aldose reductase inhibitors $[6,20]$, PKC $\beta$ inhibitors [11,12,14,21-23], and AGE inhibitors [24,25], are effective in diabetic animal models of very early DR, in which pericyte loss occurs. However, to

*Address correspondence to this author at the Department of Ophthalmology, Jichi Medical University, Saitama Medical Center, 1-847 Amanuma-cho, Omiya-ku, Saitama, 330-8503, Japan; Tel: +81-48-6472111; Fax: +81-648-5188; E-mail: kakeaki@omiya.jichi.ac.jp determine whether these drugs effectively prevent DR, an animal model of advanced DR is needed. Although numerous diabetic animal models have been described, none develops DR similar to that in humans.

The SDT rat, a substrain of the Sprague-Dawley (SD) rat, spontaneously develops diabetes mellitus and exhibits the three major diabetic ocular complications, cataracts, advanced DR, and rubeotic glaucoma. In 1988, five male rats with polyuria and glucosuria were identified among 305 rats from an outbred colony of the Crj:CD(SD) strain (Charles River Japan, Inc., Kanagawa, Japan) of SD rats. After the 20th generation of sister-brother matings, the diabetic strain was established in 1997. The characteristics of this rat have been described previously [26]. Briefly, male rats develop marked hyperglycemia (about $700 \mathrm{mg} / \mathrm{dl}$ ) and glucosuria after 20 weeks of age. The cumulative incidence of diabetes is almost $100 \%$ by 40 weeks of age. Female rats also develop diabetes but after 45 weeks of age, and the cumulative incidence is only $35 \%$ even after 60 weeks of age. The survival rates of untreated male and female SDT rats up to 65 weeks are $93 \%$ and $97 \%$, respectively. Mature diabetic cataracts are observed after 40 weeks of age in most male SDT rats. Large retinal folds mimicking tractional retinal detachment with extensive leakage of fluorescein around the 
optic disc are the most prominent finding of DR in old (mostly after 51 weeks of age) SDT rats [27]. Young SDT rats (under 50 weeks of age) usually do not exhibit the changes of advanced DR. In the current study, we performed immunohistochemical studies that focused on AGEs and VEGF in SDT rats.

\section{ANIMALS AND METHODS}

After 60 weeks of age, one eye was obtained from 10 nondiabetic normal SD rats and six SDT rats. SDT rats were confirmed to be diabetic (blood glucose level, $>250 \mathrm{mg} / \mathrm{dl}$ ). The care and handling of all animals were in accordance with the Association for Research in Vision and Ophthalmology Statement for the Use of Animals in Ophthalmic and Vision Research. Under deep anesthesia following an intraperitoneal injection of pentobarbital sodium $(30 \mathrm{mg} / \mathrm{kg}$ body weight, Nembutal, Dainihonseiyaku, Osaka, Japan), the eyes were enucleated for conventional histopathologic and immunohistochemical studies. The eyes were fixed in a mixture of $2.5 \%$ paraformaldehyde and $1 \%$ glutaraldehyde in $0.15 \mathrm{M}$ phosphate buffer (pH 7.1) to avoid artificial retinal detachment and embedded in paraffin and sectioned for immunohistochemical study of VEGF and AGEs (pyrraline, pentosidine, carboxy methyl lysine $[\mathrm{CML}])$. The immunohistochemical procedures were based on the standard avidin-biotin horseradish peroxidase method using each antibody and developed with AEC Substrate Chromogen (DakoCytomation, Carpinteria, CA, USA). VEGF was immunostained with a monoclonal antibody for human VEGF (1:25 dilution, Immuno-Biological Laboratories Co., Ltd, Fujioka, Japan). Pyrraline, pentosidine, and CML also were immunostained with a monoclonal antibody for human AGEs (1:50 dilution for pyrraline, 1:50 dilution for pentosidine, and 1:50 dilution for CML, Trans Genic Inc., Kumamoto, Japan). Bovine serum was used as a primary antibody for negative control of the immunostaining. The immunostaining grades were divided into three groups, minimal, moderate, and severe, according to the degree of staining. Minimal staining was characterized by almost no retinal staining, moderate staining by light red retinal staining, and severe staining by strong dark red retinal staining. We evaluated the grade of the immunostaining in each sample without knowing whether the eye was obtained from a SD or SDT rat.

The prevalence rates of AGEs and VEGF were evaluated using the Cochran-Armitage test and Fisher's exact test for independence. $\mathrm{P}<0.05$ was considered statistically significant.

\section{RESULTS}

Table 1 shows the prevalence rates of immunostaining for CML, pyrraline, pentosidine, and VEGF in the retinas of the diabetic rats. Fig. (1) shows immunostaining for CML, pyrraline, pentosidine, and VEGF in the retinas of SDT rats. Table 2 shows the prevalence rates of immunostaining for CML, pyrraline, pentosidine, and VEGF in the retinas of nondiabetic SD rats. Fig. (2) shows immunostaining for CML, pyrraline, pentosidine, and VEGF in the retinas of nondiabetic SD rats. The prevalence rates of immunoreactivity for CML and VEGF were significantly greater in the retinas of diabetic rats than in nondiabetic rats $(\mathrm{P}<0.05$, $\mathrm{P}<0.001$, respectively, by the Cochran-Armitage test). There was no significant difference in immunoreactivity for pyrraline and pentosidine between the diabetic and nondiabetic rats $(\mathrm{P}=0.35, \mathrm{P}=0.38$, respectively, by Fisher's exact test).

Table 1. Prevalence Rates of Immunostaining for CML, Pyrraline, Pentosidine, and VEGF in the Retinas of Diabetic Rats

\begin{tabular}{|c|c|c|c|c|}
\hline & CML & Pyrraline & Pentosidine & VEGF \\
\hline \hline Minimal & 0 & 5 & 5 & 0 \\
\hline Moderate & 1 & 1 & 1 & 3 \\
\hline Severe & 5 & 0 & 0 & 3 \\
\hline
\end{tabular}

Table 2. Prevalence Rates of Immunostaining for CML, Pyrraline, Pentosidine, and VEGF in the Retinas of Nondiabetic SD Rats

\begin{tabular}{|c|c|c|c|c|}
\hline & CML & Pyrraline & Pentosidine & VEGF \\
\hline \hline Minimal & 1 & 6 & 10 & 9 \\
\hline Moderate & 7 & 4 & 0 & 1 \\
\hline Severe & 2 & 0 & 0 & 0 \\
\hline
\end{tabular}

\section{DISCUSSION}

The most frequent ocular complications found in diabetes, i.e., cataracts, retinopathy, and neovascular glaucoma, are thought to be caused by increased polyol pathway activity [6-9], activation of PKC [10-14], increased oxidative stress $[13,15,16]$, and accumulation of AGEs as a result of prolonged hyperglycemia. In advanced cases of diabetes, retinal and iris neovascularization may occur, resulting in blindness due to proliferative DR, neovascular glaucoma, or both. VEGF is necessary for neovascularization in diabetic eyes. Increased polyol pathway activity, activation of PKC, oxidative stress, and AGEs could all induce VEGF. Although these factors can be improved with glycemic control, the AGEs are resistant to degradation and continue to accumulate in ocular tissues even in patients with diabetes with good glycemic control. Among the numerous AGEs, CML is thought to be the major one that causes ocular complications in diabetes $[13,28,29]$. However, pentosidine is more important than CML in the development of diabetic nephropathy [29-31]. Hammes et al. reported that accumulation of CML can be identified even in early-stage DR, while in the advanced stages of diabetes, accumulation of CML accompanies progression of DR [18]. Several clinical studies have shown that the level of pentosidine is more related to nephropathy than retinopathy $[29,32]$. We found that accumulation of CML was observed somewhat in nondiabetic normal SD rats. In SDT rats, staining for CML was stronger than in nondiabetic rats, and the prevalence of VEGF was greater than in nondiabetic rats. However, there was no significant difference in the prevalence of pyrraline and pentosidine between diabetic rats 


\section{Pyrraline, minimal}

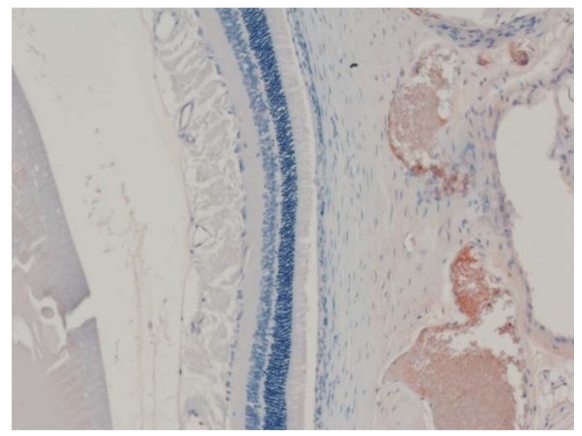

Pentosidine, minimal

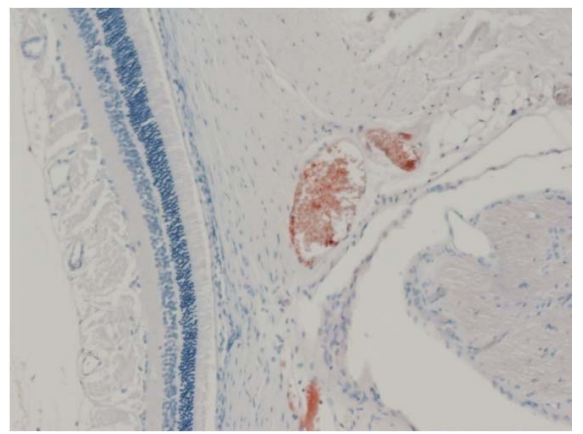

\section{$\mathrm{CML}$, severe}

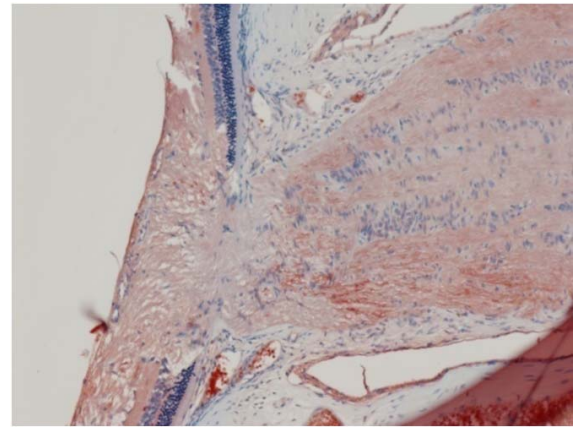

VEGF, severe

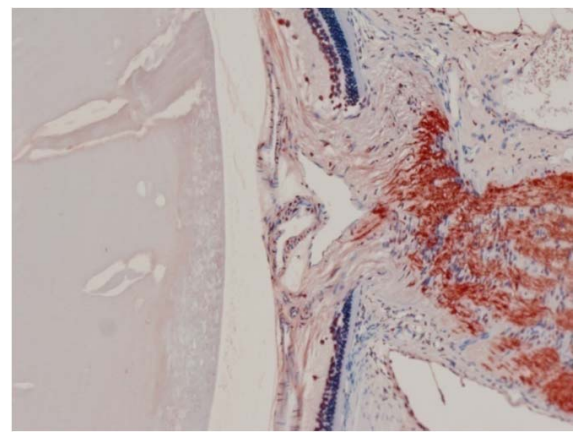

Fig. (1). Immunostaining for AGEs and VEGF in the retinas of diabetic SDT rats. CML and VEGF are clearly seen, but pyrraline and pentosidine are not (original magnification $\mathrm{x} 10$ ).

Pyrraline, minimal

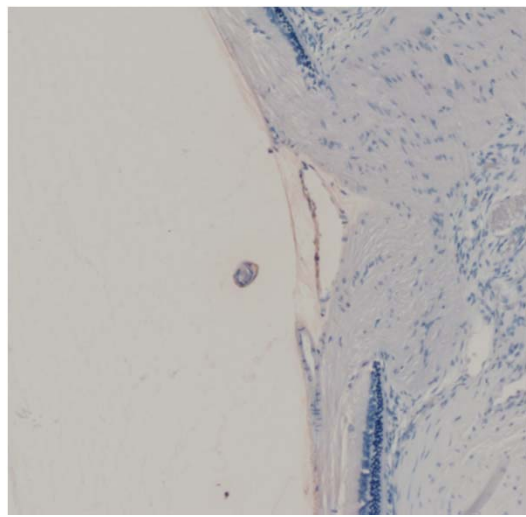

Pentosidine, minimal

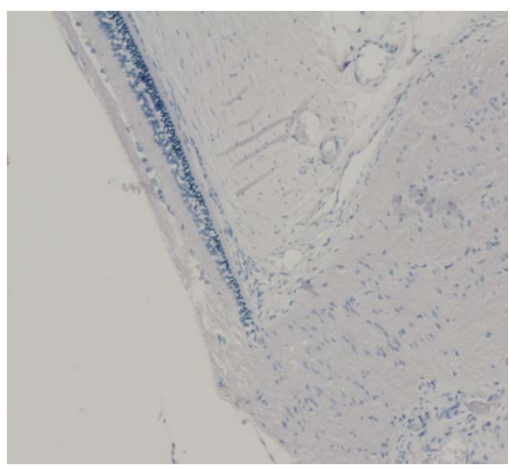

CML, moderate

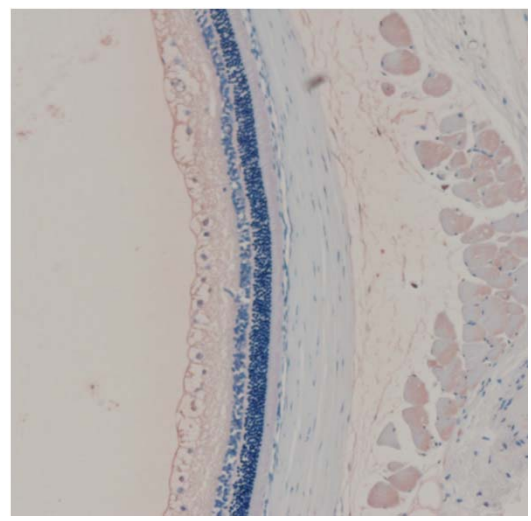

VEGF, minimal

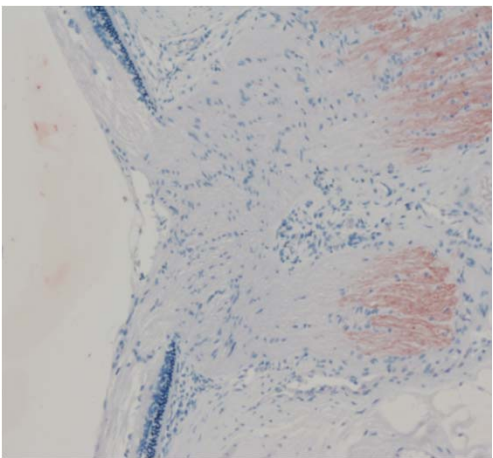

Fig. (2). Immunostaining for AGEs and VEGF in the retinas of nondiabetic SD rats. Staining for CML is weaker than in diabetic SDT rats. Pyrraline, pentosidine, and VEGF are not clearly seen (original magnification x10). 
and nondiabetic rats. Therefore, VEGF expression was correlated with CML expression but not pentosidine or pyrraline. It appears that CML may be the most important AGE-inducing neovascularization in the eye, while pyrraline and pentosidine are contributory. Since the relationship of AGEs to diabetic ocular complications in SDT rats closely resembles that in humans, the SDT rat may be a useful animal model for investigating the pathogenesis of diabetic ocular complications.

\section{ACKNOWLEDGMENTS}

We thank Dr. Wilfred Y Fujimoto for helpful suggestions and Ms. Kimiko Aoki for technical assistance in the histopathologic studies.

\section{REFERENCES}

[1] Hyman L. Epidemiology of eye disease in the elderly. Eye 1987; 1 (Pt 2): 330-41.

[2] Krumpaszky HG, Ludtke R, Mickler A, Klauss V, Selbmann HK. Blindness incidence in Germany. A population-based study from Wurttemberg-Hohenzollern. Ophthalmologica 1999; 213: 176-82.

[3] Kocur I, Resnikoff S. Visual impairment and blindness in Europe and their prevention. Br J Ophthalmol 2002; 86: 716-22.

[4] Aiello LP, Avery RL, Arrigg PG, et al. Vascular endothelial growth factor in ocular fluid of patients with diabetic retinopathy and other retinal disorders. N Engl J Med 1994; 331: 1480-7.

[5] Adamis AP, Miller JW, Bernal MT, et al. Increased vascular endothelial growth factor levels in the vitreous of eyes with proliferative diabetic retinopathy. Am J Ophthalmol 1994; 118: 445-50.

[6] Robison WG, Jr., Nagata M, Tillis TN, Laver N, Kinoshita JH. Aldose reductase and pericyte-endothelial cell contacts in retina and optic nerve. Invest Ophthalmol Vis Sci 1989; 30: 2293-9.

[7] Hotta N, Nakamura J, Sakakibara F, et al. Electroretinogram in sucrose-fed diabetic rats treated with an aldose reductase inhibitor or an anticoagulant. Am J Physiol 1997; 273: E965-971.

[8] Miwa K, Nakamura J, Hamada Y, et al. The role of polyol pathway in glucose-induced apoptosis of cultured retinal pericytes. Diabetes Res Clin Pract 2003; 60: 1-9.

[9] Dagher Z, Park YS, Asnaghi V, Hoehn T, Gerhardinger C, Lorenzi M. Studies of rat and human retinas predict a role for the polyol pathway in human diabetic retinopathy. Diabetes 2004; 53: 240411.

[10] Lee TS, MacGregor LC, Fluharty SJ, King GL. Differential regulation of protein kinase $\mathrm{C}$ and $(\mathrm{Na}, \mathrm{K})$-adenosine triphosphatase activities by elevated glucose levels in retinal capillary endothelial cells. J Clin Invest 1989; 83: 90-4.

[11] Aiello LP, Bursell SE, Clermont A, et al. Vascular endothelial growth factor-induced retinal permeability is mediated by protein kinase $\mathrm{C}$ in vivo and suppressed by an orally effective betaisoform-selective inhibitor. Diabetes 1997; 46: 1473-80.

[12] Nakamura J, Kato K, Hamada Y, et al. A protein kinase C-betaselective inhibitor ameliorates neural dysfunction in streptozotocininduced diabetic rats. Diabetes 1999; 48: 2090-5.

[13] Hammes HP, Brownlee M, Lin J, Schleicher E, Bretzel RG. Diabetic retinopathy risk correlates with intracellular concentrations of the glycoxidation product Nepsilon(carboxymethyl) lysine independently of glycohaemoglobin concentrations. Diabetologia 1999; 42: 603-7.

[14] Aiello LP. The potential role of PKC beta in diabetic retinopathy and macular edema. Surv Ophthalmol 2002; 47 (suppl 2): S263-9.

[15] Kuroki M, Voest EE, Amano S, et al. Reactive oxygen intermediates increase vascular endothelial growth factor expression in vitro and in vivo. J Clin Invest 1996; 98: 1667-75.
[16] Altomare E, Grattagliano I, Vendemaile G, Micelli-Ferrari T, Signorile A, Cardia L. Oxidative protein damage in human diabetic eye: evidence of a retinal participation. Eur J Clin Invest 1997; 27: 141-7.

[17] Murata T, Nagai R, Ishibashi T, Inomuta H, Ikeda K, Horiuchi S. The relationship between accumulation of advanced glycation end products and expression of vascular endothelial growth factor in human diabetic retinas. Diabetologia 1997; 40: 764-9.

[18] Hammes HP, Alt A, Niwa T, et al. Differential accumulation of advanced glycation end products in the course of diabetic retinopathy. Diabetologia 1999; 42: 728-36.

[19] Hammes HP, Wellensiek B, Kloting I, Sickel E, Bretzel RG, Brownlee M. The relationship of glycaemic level to advanced glycation end-product (AGE) accumulation and retinal pathology in the spontaneous diabetic hamster. Diabetologia 1998; 41: 16570 .

[20] Robison WG, Jr., Nagata M, Laver N, Hohman TC, Kinoshita JH. Diabetic-like retinopathy in rats prevented with an aldose reductase inhibitor. Invest Ophthalmol Vis Sci 1989; 30: 2285-92.

[21] Nonaka A, Kiryu J, Tsujikawa A, et al. PKC-beta inhibitor (LY333531) attenuates leukocyte entrapment in retinal microcirculation of diabetic rats. Invest Ophthalmol Vis Sci 2000; 41: $2702-6$.

[22] The effect of ruboxistaurin on visual loss in patients with moderately severe to very severe nonproliferative diabetic retinopathy: initial results of the Protein Kinase $\mathrm{C}$ beta Inhibitor Diabetic Retinopathy Study (PKC-DRS) multicenter randomized clinical trial. Diabetes 2005; 54: 2188-97.

[23] Aiello LP, Clermont A, Arora V, Davis MD, Sheetz MJ, Bursell SE. Inhibition of PKC beta by oral administration of ruboxistaurin is well tolerated and ameliorates diabetes-induced retinal hemodynamic abnormalities in patients. Invest Ophthalmol Vis Sci 2006; 47: 86-92.

[24] Hammes HP, Martin S, Federlin K, Geisen K, Brownlee M. Aminoguanidine treatment inhibits the development of experimental diabetic retinopathy. Proc Natl Acad Sci U S A 1991; 88: $11555-8$

[25] Hammes HP, Strodter D, Weiss A, Bretzel RG, Federlin K, Brownlee M. Secondary intervention with aminoguanidine retards the progression of diabetic retinopathy in the rat model. Diabetologia 1995; 38: 656-60.

[26] Shinohara M, Masuyama T, Shoda T, et al. A new spontaneously diabetic non-obese Torii rat strain with severe ocular complications. Int J Exp Diabetes Res 2000; 1: 89-100.

[27] Kakehashi A, Saito Y, Mori K, et al. Characteristics of diabetic retinopathy in SDT Rats. Diabetes Metab Res Rev 2006; 22: 45561.

[28] Boehm BO, Schilling S, Rosinger S, et al. Elevated serum levels of $\mathrm{N}$ (epsilon)-carboxymethyl-lysine, an advanced glycation end product, are associated with proliferative diabetic retinopathy and macular oedema. Diabetologia 2004; 47: 1376-79.

[29] Hirata K, Kubo K. Relationship between blood levels of Ncarboxymethyl-lysine and pentosidine and the severity of microangiopathy in type 2 diabetes. Endocr J 2004; 51: 537-44.

[30] Sugiyama S, Miyata T, Horie K, et al. Advanced glycation endproducts in diabetic nephropathy. Nephrol Dial Transplant 1996; 11 (suppl 5): 91-4.

[31] Weiss MF, Rodby RA, Justice AC, Hricik DE. Free pentosidine and neopterin as markers of progression rate in diabetic nephropathy. Collaborative Study Group. Kidney Int 1998; 54: 193-202.

[32] Capeans Tome C, De Rojas Silva MV, Rodriguez-Garcia J, Rodriguez-Segade S, Sanchez-Salorio M. Levels of pentosidine in the vitreous of eyes with proliferative diabetic retinopathy, proliferative vitreoretinopathy and retinal detachment. Graefes Arch Clin Exp Ophthalmol 2005; 243: 1272-6. 